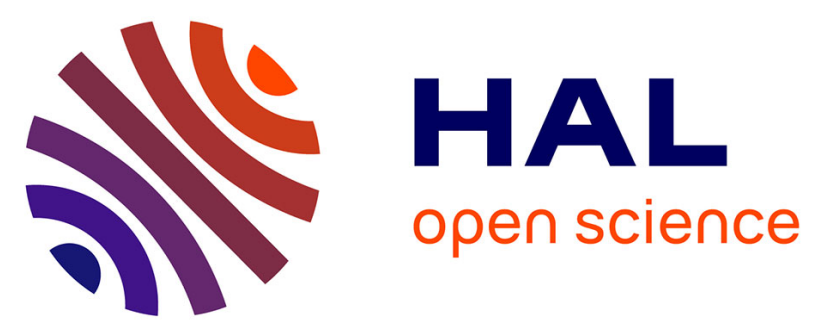

\title{
Complexity of the Ruminococcus flavefaciens cellulosome reflects an expansion in glycan recognition
} Immacolata Venditto, Ana S. Luis, Maja Rydahl, Julia Schuckel, Vania O. Fernandes, Silvia Vidal-Melgosa, Pedro Bule, Arun Goyal, Virginia M. R. Pires, Catarina G. Dourado, et al.

\section{To cite this version:}

Immacolata Venditto, Ana S. Luis, Maja Rydahl, Julia Schuckel, Vania O. Fernandes, et al.. Complexity of the Ruminococcus flavefaciens cellulosome reflects an expansion in glycan recognition. Proceedings of the National Academy of Sciences of the United States of America, 2016, 113 (26), pp.7136-7141. 10.1073/pnas.1601558113 . hal-01439108

\section{HAL Id: hal-01439108 https://hal.science/hal-01439108}

Submitted on 28 May 2020

HAL is a multi-disciplinary open access archive for the deposit and dissemination of scientific research documents, whether they are published or not. The documents may come from teaching and research institutions in France or abroad, or from public or private research centers.
L'archive ouverte pluridisciplinaire HAL, est destinée au dépôt et à la diffusion de documents scientifiques de niveau recherche, publiés ou non, émanant des établissements d'enseignement et de recherche français ou étrangers, des laboratoires publics ou privés.

\section{(ㅇ)(1) $\$$}

Distributed under a Creative Commons Attribution - NonCommerciall 4.0 International 


\title{
Complexity of the Ruminococcus flavefaciens cellulosome reflects an expansion in glycan recognition
}

\author{
Immacolata Venditto ${ }^{a, b, 1}$, Ana S. Luis ${ }^{a, b, 1}$, Maja Rydahlc, ${ }^{c, 1}$, Julia Schückele,1, Vânia O. Fernandes ${ }^{a, d}$, Silvia Vidal-Melgosac, \\ Pedro Bule $^{a}$, Arun Goyale, Virginia M. R. Pires ${ }^{a}$, Catarina G. Dourado a, Luís M. A. Ferreira a,d, Pedro M. Coutinhof, \\ Bernard Henrissat ${ }^{f, g, h}$, J. Paul Knox ${ }^{i}$, Arnaud Basléb ${ }^{b}$, Shabir Najmudina ${ }^{\text {, Harry J. Gilbert }}{ }^{b, 2}$, William G. T. Willats ${ }^{c, 2}$, \\ and Carlos M. G. A. Fontes ${ }^{\mathrm{a}, \mathrm{d}, 2}$

\begin{abstract}
a Interdisciplinary Centre of Research in Animal Health, Faculdade de Medicina Veterinária, Universidade de Lisboa, Pólo Universitário do Alto da Ajuda, 1300-477 Lisbon, Portugal; 'bnstitute for Cell and Molecular Biosciences, Newcastle University, Newcastle upon Tyne NE2 4HH, United Kingdom;

'Department of Plant and Environmental Sciences, Faculty of Science, University of Copenhagen, Copenhagen, Denmark; ${ }^{d}$ NZYTech Genes \& Enzymes, Campus do Lumiar, 1649-038 Lisbon, Portugal; ' ${ }^{2}$ epartment of Biotechnology, Indian Institute of Technology Guwahati, Guwahati, Assam, India; ${ }^{\mathrm{f}}$ Architecture et Fonction des Macromolécules Biologiques, UMR 7857 CNRS, Aix-Marseille University, F-13288 Marseille, France; ${ }^{9}$ Institut National de la Recherche Agronomique, USC 1408 Architecture et Fonction des Macromolécules Biologiques, F-13288 Marseille, France, ${ }^{\text {h}}$ Department of Biological
\end{abstract} \\ Sciences, King Abdulaziz University, Jeddah, Saudi Arabia; and 'Centre for Plant Sciences, University of Leeds, Leeds LS2 9JT, United Kingdom
}

Edited by Arnold L. Demain, Drew University, Madison, NJ, and approved May 6, 2016 (received for review January 28, 2016)

The breakdown of plant cell wall (PCW) glycans is an important biological and industrial process. Noncatalytic carbohydrate binding modules (CBMs) fulfill a critical targeting function in PCW depolymerization. Defining the portfolio of CBMs, the CBMome, of a PCW degrading system is central to understanding the mechanisms by which microbes depolymerize their target substrates. Ruminococcus flavefaciens, a major PCW degrading bacterium, assembles its catalytic apparatus into a large multienzyme complex, the cellulosome. Significantly, bioinformatic analyses of the $R$. flavefaciens cellulosome failed to identify a CBM predicted to bind to crystalline cellulose, a key feature of the CBMome of other PCW degrading systems. Here, high throughput screening of 177 protein modules of unknown function was used to determine the complete CBMome of $\boldsymbol{R}$. flavefaciens. The data identified six previously unidentified CBM families that targeted $\beta$-glucans, $\beta$-mannans, and the pectic polysaccharide homogalacturonan. The crystal structures of four CBMs, in conjunction with site-directed mutagenesis, provide insight into the mechanism of ligand recognition. In the CBMs that recognize $\beta$-glucans and $\beta$-mannans, differences in the conformation of conserved aromatic residues had a significant impact on the topology of the ligand binding cleft and thus ligand specificity. A cluster of basic residues in CBM77 confers calcium-independent recognition of homogalacturonan, indicating that the carboxylates of galacturonic acid are key specificity determinants. This report shows that the extended repertoire of proteins in the cellulosome of $R$. flavefaciens contributes to an extended CBMome that supports efficient PCW degradation in the absence of CBMs that specifically target crystalline cellulose.

carbohydrate-binding modules | protein-carbohydrate interactions | carbohydrate active enZYmes | cellulosomes

Dlant cell walls (PCWs) consist of interlinked polysaccharides, often impregnated with lignin that evolved to restrict access to enzyme attack. Thus, the recycling of photosynthetically fixed carbon is a slow biological process. Reflecting the intricacy of PCWs, microorganisms that degrade these composite structures produce extensive repertoires of carbohydrate active enzymes (CAZymes) (1), which are of increasing industrial significance (2).

CAZymes acting on recalcitrant carbohydrates are frequently appended with noncatalytic carbohydrate binding modules (CBMs). CBMs potentiate the activity of the associated catalytic modules through substrate targeting (see ref. 3 for a review). CBMs and CAZymes are classified into sequence-based families in the CAZy database (www.cazy.org/) (4). Based on their binding mode, CBMs have been classified into three types. Type A CBMs display a planar surface that binds to crystalline polysaccharides; type B modules accommodate internal regions of glycan chains within open clefts; and type C CBMs recognize the termini of glycans (exo-type) in a binding site that adopts a pocket topology (3).

Efficient hydrolysis of PCW polysaccharides has been finetuned over millions of years in ecological niches that are subjected to intensive selective pressures exemplified by the rumen of mammalian herbivores. A cohort of rumen anaerobic bacteria assemble their PCW degrading apparatus into multiprotein complexes termed cellulosomes (5). Cellulosome assembly is through the interaction of cohesin modules located on the noncatalytic protein, the scaffoldin, and dockerin modules on each enzyme subunit (5). Clostridial cellulosomes bind tightly to PCWs through a scaffoldin family $3 \mathrm{CBM}$. The repertoire of cellulosomal enzymes expressed by an individual bacterium constitutes a highly selected consortium of biocatalysts optimized to degrade PCWs. Genome sequencing of Ruminococcus flavefaciens strain FD-1 (6), the most abundant ruminal cellulolytic bacterium, revealed an elaborate assembly of scaffoldins, indicating

\section{Significance}

Plant cell wall (PCW) polysaccharide degradation is an important biological and industrial process. Noncatalytic carbohydrate binding modules (CBMs) fulfill a critical targeting function in PCW depolymerization. Ruminococcus flavefaciens synthesizes a highly efficient PCW degrading apparatus. Here, six previously unidentified $R$. flavefaciens CBM families were identified that targeted $\beta$-glucans, $\beta$-mannans, and pectins. Crystal structures of these CBMs revealed that recognition of $\beta$-glucans and $\beta$-mannans was mediated by differences in the conformation of conserved aromatic residues in the ligand binding cleft. A cluster of basic residues in CBM77 confers calcium-independent recognition of homogalacturonan. This report shows that the expansion of protein modules in the cellulosome of $\boldsymbol{R}$. flavefaciens contributes to an extended CBM profile that supports efficient PCW degradation.

Author contributions: A.S.L., L.M.A.F., P.M.C., B.H., A.B., S.N., H.J.G., W.G.T.W, and C.M.G.A.F. designed research; I.V., A.S.L., M.R., J.S., V.O.F., S.V.-M., P.B., A.G., V.M.R.P., C.G.D., A.B., and S.N. performed research; I.V., A.S.L., M.R., J.S., V.O.F., S.V.-M., P.B., A.G., V.M.R.P., C.G.D. L.M.A.F., P.M.C.,B.H., J.P.K., A.B., S.N., W.G.T.W, and C.M.G.A.F. analyzed data; and I.V., H.J.G., W.G.T.W, and C.M.G.A.F. wrote the paper.

The authors declare no conflict of interest.

This article is a PNAS Direct Submission.

Freely available online through the PNAS open access option.

Data deposition: The atomic coordinates and structure factors have been deposited in the Protein Data Bank, www.pdb.org (PDB ID codes 5AOT, 5FU2, 5FU3, 5FU4, 5FU5, 4VI7, and 4VIL).

${ }^{1}$ I.V., A.S.L., M.R., and J.S. contributed equally to this work.

${ }^{2}$ To whom correspondence may be addressed. Email: cafontes@fmv.ulisboa.pt, willats@ plen.ku.dk, or harry.gilbert@ncl.ac.uk.

This article contains supporting information online at www.pnas.org/lookup/suppl/doi:10 1073/pnas.1601558113/-/DCSupplemental. 
that the bacterium's cellulosome is an intricate and versatile PCW degrading system. Commensurate with this proposed cellulosomal complexity, the genome of $R$. flavefaciens FD-1 encodes $\sim 230$ dockerin-containing proteins, which are likely to integrate into the multienzyme complex (6). A large number of the protein modules identified in the $R$. flavefaciens cellulosome are of unknown function and may reflect an extended capacity to recognize carbohydrates through an extended CBM profile.

One of the major challenges facing postgenomic analysis of organisms is the identification of the function of the large number of predicted proteins derived from genomic sequencing. To bridge this gap in knowledge requires the development of high throughput methodologies (HTPMs). Here, we have explored how HTPMs can be used to interrogate the functional complexity of the $R$. flavefaciens cellulosome. The data support the hypothesis that protein diversity in the $R$. flavefaciens cellulosome contributes to an expansion in glycan recognition, which is mediated by a ruminococcal-specific cohort of protein modules.

\section{Results and Discussion}

R. flavefaciens Cellulosomal Enzymes Contain Previously Unidentified CBMs. The $R$. flavefaciens FD-1 cellulosome contains 177 protein modules of unknown function (UNKs). These UNKs were assessed for CBM functions using a carbohydrate microarray platform that enabled rapid screening of binding against multiple glycans. The microarrays were populated with 18 oligosaccharides and 46 polysaccharides of PCW origin (7). The output from the microarrays identified nine CBMs that bound to at least one arrayed glycan (Fig. 1A) (nonbinding glycans are listed in Table $\mathrm{S} 1)$. The positive hits were also screened by affinity gel electrophoresis (AGE) (Fig. $1 B$ and Fig. S1 $A$ ), and the assignment of ligand specificity described below in this section and in Fig. 2 was derived from these AGE experiments. Based on sequence similarity, the nine CBMs were grouped into six previously unidentified families designated CBM75 to CBM80. The protein modules were defined by the CBM family (CBMXX) and enzyme (RfGHXX) from which they were derived. Although the C-terminal portion of $\mathrm{CBM} 75_{\mathrm{RfGH}_{43}}$ displayed distant sequence similarity to members of CBM6, this region of the protein was not responsible for ligand recognition (SI Results), and thus the protein module was designated as a novel CBM family. An overview of the specificity of the six novel CBM families is as follows: CBM75 is a xyloglucan-specific family; CBM76 recognized different $\beta$-1,4-glucans; and CBM79 bound to a range of $\beta-1,4-$ and mixed linked $\beta-1,3-1,4$-glucans. Similarly, families CBM78 and CBM80 displayed specificity for $\beta-1,4-$ and mixed linked $\beta-1,3-1,4$-glucans, with some members also binding to $\beta-1,4-$ mannans. Thus, in CBM78 and CBM80, the proteins
$\mathrm{CBM} 78_{\mathrm{GH} 26}$ and $\mathrm{CBM} 80_{\mathrm{RfGH} 5-1 / 2}$, respectively, bound to galactomannan in addition to the $\beta$-glucans whereas CBM78 $8_{\mathrm{RfGH} 5}$ and $\mathrm{CBM} 80_{\mathrm{RfGH} 5}$ recognized only the gluco-configured ligands. None of the $\beta$-glucan binding CBMs bound to $\beta$-1,3-glucans, $\beta$-1,6-glucans, or xylans. CBM77 $7_{\mathrm{PL} 1 / 9}$ bound exclusively to homopolygalacturonan (pectin) with low degrees of methyl esterification (DEs) in vitro, and to pectin within the context of intact PCWs (Fig. 1C).

The glycan microarray and AGE data were broadly similar although subtle differences in specificity were evident. Thus, the binding of $\mathrm{CBM} 78_{\mathrm{RfGH} 5}$ to barley $\beta$-glucan was observed only using AGE whereas only microarray data revealed an interaction between $\mathrm{CBM} 75_{\mathrm{RfGH}_{43}}$ and this glycan. Such differences in specificity between the two methods may reflect variations in the conformation of some glycans arrayed on nitrocellulose or contained within polyacrylamide gels.

The Enzyme Context of the Previously Unidentified CBMs. The CBMs that bound $\beta$-glucans are components of enzymes that contain catalytic modules derived from GH5_4 (CBM78 ${ }_{\mathrm{RfGH} 5}, \mathrm{CBM} 80_{\mathrm{RfGH} 5-1 / 2}$, and $\mathrm{CBM} 80_{\mathrm{RfGH}}$ ), GH9 (enzyme contains two CBM79s), or GH44 $\left(\mathrm{CBM} 76_{\mathrm{RfGH} 44}\right)$, families/subfamilies that are populated exclusively by endo- $\beta 1$,4-glucanases (Fig. 2). The two enzymes containing CBMs that bind galactomannan have GH5 7 or GH26 " $\beta 1,4$-mannanase" catalytic modules. Indeed, the dual specificity of CBM80 ${ }_{\mathrm{RfGH} 5-1 / 2}$ is consistent with the catalytic modules of the enzymes that hydrolyze $\beta$-glucans (GH5 4) or $\beta$-mannans (GH5 7). Consistent with its specificity, $\mathrm{CBM} \overline{7} 7_{\mathrm{RfPL} 1 / 9}$ is a component of an enzyme that contains two catalytic modules, belonging to polysaccharide lyase families 1 and 9 (PL1 and PL9), which display pectate lyase activity $\left(k_{\text {cat }} / K_{\mathrm{m}}\right.$ values of PL1 and PL9 against homogalacturonan were $3.1 \times 10^{3}$ and $3.7 \times 10^{5} \mathrm{~min}^{-1} \mathrm{mg}^{-1} \mathrm{~mL}$, respectively). CBM75 ${ }_{\text {RfGH43 }}$, which binds xyloglucan, is associated with a GH43 16 catalytic module, a subfamily that, to date, contains only arabinofuranosidases (8). The GH43 catalytic module of the enzyme hydrolyzed only 4-nitrophenyl- $\alpha$-L-arabinofuranose, demonstrating that the enzyme is an arabinofuranosidase. The enzyme was not active against arabinoxylans and arabinans. CBMs generally display specificities consistent with the activity of the appended enzyme (3) although glycan recognition can be at the interface between the two modules, illustrated by the arabinoxylan binding function of a modular arabinofuranosidase (9). Given that CBM75 ${ }_{\text {RfGH43 }}$ bound to xyloglucan, we speculate that the GH43 16 targets arabinofuranose residues that decorate xyloglucans from tomato (10).

Phylogeny of the Six Previously Unidentified CBM Families. Representatives of the six novel Ruminococcus CBM families were
A

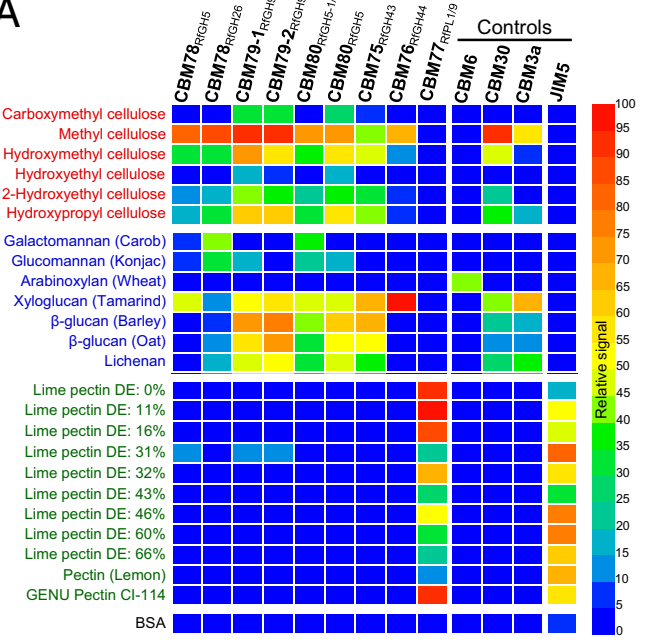

B

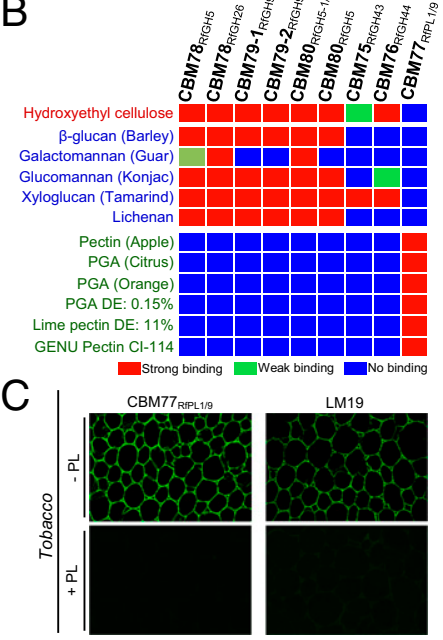

Fig. 1. Screening $R$. flavefaciens UNKs for glycan binding functions. (A) Glycan microarray binding profiles of the founding members of six previously unidentified CBM families. CBM6, -CBM30, and CBM3a (characterized CBMs) and JIM5, a monoclonal antibody (mAb) that binds homogalacturonan, were used as binding controls. The mean spot signals obtained from two individual experiments are presented in a heat map in which color intensity is correlated to signal. The highest signal in the dataset was set to 100 , and all other values were normalized accordingly (in accordance with the color intensity scale bar). Glycans that did not bind to any of the proteins screened are listed in Table S1. (B) Binding affinity of different CBMs detected by AGE. Red signifies binding, light green signifies marginal binding, and blue represents no detectable binding. (C) Evaluation of the binding of CBM77 $7_{\mathrm{RPP} L 1 / 9}$ to pectic homogalacturonan in tobacco stem sections. LM19 is an mAb that binds unesterified homogalacturonan. The binding capacity of CBM77 $7_{\mathrm{RfPL} 1 / 9}$ and LM19 was evaluated before and after section pretreatment with a pectate lyase. 


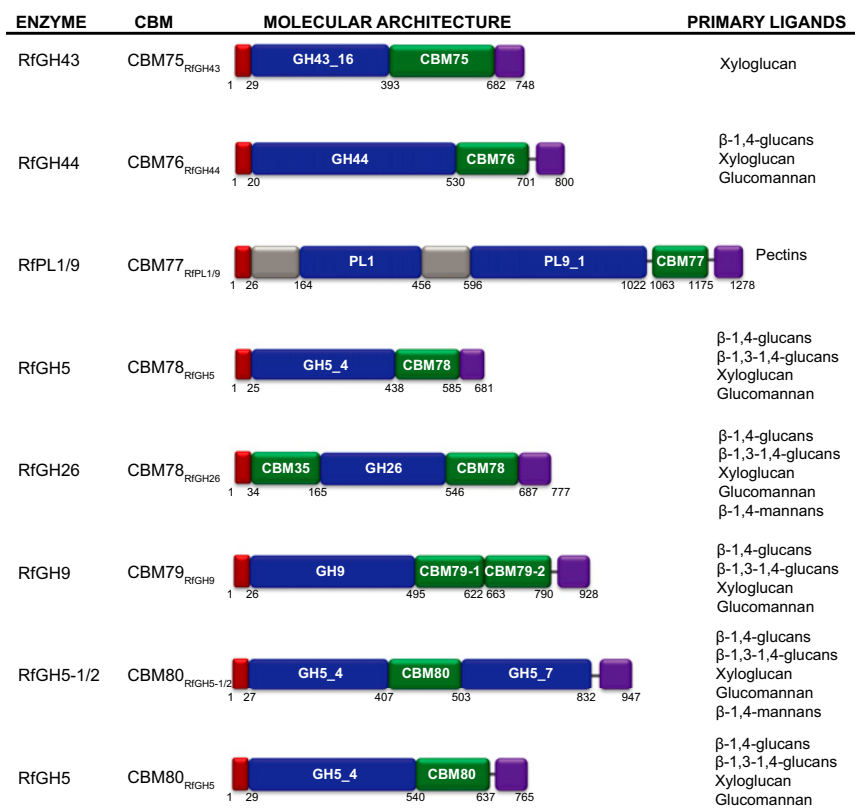

Fig. 2. Molecular architectures of proteins containing previously unidentified CBMs identified in this study. The origins of the families of glycoside hydrolases (GHs) and polysaccharide lyases (PLs) are identified. The modules of unknown function are colored gray, signal peptides red, and dockerin modules purple. Linker sequences are depicted by a line. The boundaries of the modules in the full-length sequence of the enzymes are indicated. The polysaccharides targeted by the different CBMs are indicated.

used in a BLAST search of the NCBI protein sequence database. Sequences were retrieved with $E$ values $<4 \times 10^{-4}$ with sequence identify $>30 \%$. No sequence corresponding to a CBM annotated on the CAZy database (4) was identified, confirming the discovery of six previously unidentified CBM families (Fig. S2). Families CBM75-76 and CBM78-80 contained sequences derived exclusively from ruminococci. In contrast, the catalytic modules appended to these CBMs displayed sequence similarity to glycoside hydrolases from a range of prokaryotes. These data indicate that the catalytic modules of the $R$. flavefaciens enzymes were acquired through horizontal gene transfer and subsequently appended with the Ruminococcus-specific CBMs. CBM77 contains $\sim 140$ members from a range of bacteria. In the majority of the nonruminococci proteins, the CBM77 is appended to PL1 catalytic modules although PL9 sequences were also present in a cohort of enzymes (Fig. S3). Based on the phylogeny, it would seem that CBM75, $-76,-78,-79$, and -80 fulfill an enzyme-targeting role that is specific to Ruminococcus. It is possible that the contribution of these CBMs to enzyme function is evident only in a highly complex scaffold provided by the intricate organization of the $R$. flavefaciens cellulosome. In contrast, the CBM77 seems to play a more general role in pectin degradation that is not specific to ruminococci or cellulosome organization.

Thermodynamics of Ligand Binding for Selected CBMs. The affinity of representatives of the six previously unidentified CBM families for their respective ligands was determined by isothermal titration calorimetry (ITC) (Table 1 and Fig. S1B, with thermodynamic parameters reported in Tables $\mathrm{S} 2-\mathrm{S} 7$ ). CBM75 ${ }_{\mathrm{GH} 43}$ bound exclusively to xyloglucan. The affinity of the CBM for the oligosaccharide XXXG (X, glucose linked O6 to xylose; G, undecorated glucose), the repeating unit of xyloglucan, was similar to the polysaccharide (Table 1). This finding indicates that the protein contains four binding sites that interact with the backbone glucose units and at least some of the xylose side chains.

$\mathrm{CBM} 76_{\mathrm{GH} 44}$ and $\mathrm{CBM} 78_{\mathrm{GH} 5}$ displayed highest affinity for xyloglucan. The similar $K_{\mathrm{A}}$ values of $\mathrm{CBM} 78_{\mathrm{GH} 5}$ for cellohexaose and cellopentaose suggested five dominant sugar binding sites. The affinity of $\mathrm{CBM} 78_{\mathrm{GH} 5}$ for $\mathrm{XXXG}$ was significantly higher than cellotetraose (Table 1), suggesting that recognition of the xylose side chains occurred within the core binding sites of the protein.

CBM77 ${ }_{\mathrm{PL} 1 / 9}$ displayed high affinity for low DE pectins (Table 1). Only oligosaccharides with a degree of polymerization (DP) $\geq 7$ bound to the CBM, indicating that the binding site interacts with seven or eight GalA residues. Ligand recognition by CBM77 $7_{\mathrm{PL} 1 / 9}$ was not inhibited by EDTA, indicating that pectin binding was metal-independent, which is in contrast to pectate lyases, where calcium is a central feature of GalA recognition. CBM32 from Yersinia (11), which is not a component of an enzyme, is the only other example of a CBM that binds to pectin backbones. The CBM32 binds optimally to $\sim 10 \mathrm{GalA}$ residues although the role of metal ions in ligand recognition was not reported.

CBM79- $1_{\mathrm{GH} 9}(\mathrm{~N}$-terminal CBM79) bound to barley $\beta$-glucan and hydroxyethylcellulose (HEC) with similar affinities. The small increase in $K_{\mathrm{A}}$ from cellotetraose to cellohexaose (Table 1) suggests that ligand recognition is again dominated by four tandem sugar binding sites. Binding to xyloglucan was weaker than the other $\beta$-glucans, indicating that the protein cannot easily accommodate the xylose side chains of the hemicellulose.

CBM $80_{\mathrm{GH}-1 / 2}$ bound to $\beta$-glucans and galactomannan with affinities in the range of $10^{4}$ to $10^{5} \mathrm{M}^{-1}$ (Table 1 ). The protein bound to cellulooligosaccharides and mannooligosaccharides with a DP of 5 or 6 with similar affinities. Although $\mathrm{CBM} 80_{\mathrm{GH}-1 / 2}$ bound to mannotetraose, the protein did not bind to cellotetraose. Thus, the binding region of $\mathrm{CBM} 80_{\mathrm{GH}-1 / 2}$ for the gluco-configured ligands is more extensive than for the mannose-based glycans (see below in the next section).

Crystal Structures and Ligand Recognition of Representatives of

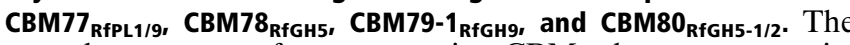
crystal structures of representative $\mathrm{CBMs}$ that target pectin, $\beta$-glucans, and mannan $/ \beta$-glucan, respectively, were solved, to a resolution of $1.5 \AA$ (CBM77 $\left.{ }_{\mathrm{RfPL} 16}\right), 2.0 \AA\left(\mathrm{CBM} 78_{\mathrm{RfGH} 5}\right), 1.8 \AA$ (CBM79-1 RfGH9 $_{1}$, and 1.0-1.5 $\AA$ (CBM80 $\left.0_{\mathrm{RfGH} 5-1 / 2}\right)$ (structure statistics are in Table S8). All of the proteins adopt a $\beta$-sandwich fold (Fig. 3) typical of CBM families (3). The $R$. flavefaciens proteins displayed 3D structural similarity, but very low sequence identity (3-11\%), to proteins in other CAZy CBM families (Table S9). There is, however, no conservation in the ligand binding residues between the Ruminococcus proteins and the structural homologs in the other CAZy CBM families. Details of the secondary structures of the CBMs are provided in Fig. S4 and the structural basis for ligand recognition are as follows.

CBM77 RfPL1/9. $_{\text {CBM77 }}$ RfPL1/9 contains two $\beta$-sheets defined as 1 and 2 (Fig. $3 D$ and Fig. S4). The canonical ligand binding site in endoacting type $\mathrm{B}$ CBMs comprises the concave surface presented by $\beta$-sheet 2 (3). In CBM77 $7_{\text {RfPL } 1 / 9}$, however, $\beta$-sheet 2 did not display this classic cleft topology, and mutation of aromatic and basic residues in the concave surface had no effect on ligand binding (Table S5). The surface of CBM77 $7_{\text {RfPL1/9 }}$ (Table S9), comprising the loops connecting the $\beta$-sheets, contains indentations. At the center of this surface are Lys ${ }^{1092}$, Lys ${ }^{1107}$, and Lys ${ }^{1162}$. Alanine substitution of these residues abrogated pectin, showing that these amino acids constitute the ligand binding site (Table S5). Distal to the central basic ligand binding site are two additional lysine residues, Lys ${ }^{1136}$ and Lys ${ }^{1141}$. The double mutant K1136A/K1141A displayed no binding to pectin although the individual mutants K1136A and K1141A retained affinity for pectin (Table S5). Lys ${ }^{1136}$ and Lys ${ }^{1141}$ may bind pectin only when the polysaccharide is in one of its two possible orientations, explaining the functional redundancy. The ligand binding surface is $\sim 25 \AA$. Pectic homogalacturonan adopts a compressed "accordion-like" structure in which a disaccharide subunit spans a distance of $8 \AA$ (11), suggesting that the binding site can accommodate a hexasaccharide. The ligand binding mode of CBM7 $77_{\mathrm{RfPL} 1 / 9}$ is distinct from other CBMs, where aromatic residues dominate glycan recognition (3), but resembles glycosaminoglycan binding proteins, where ligand recognition is also mediated by basic residues (12). Within the CBM77 family, the three 
Table 1. The affinity of CBMs for their ligands

\begin{tabular}{|c|c|c|}
\hline CBM & Ligand & $K_{\mathrm{A}}, \mathrm{M}^{-1}$ \\
\hline \multirow[t]{5}{*}{ CBM75 $5_{\text {RfGH43 }}$} & Xyloglucan & $1.7( \pm 0.2) \times 10^{4}$ \\
\hline & Glucomannan & No binding \\
\hline & $\beta$-Glucan & No binding \\
\hline & HEC & Binding weak* \\
\hline & XXXG & $4.0( \pm 0.6) \times 10^{3}$ \\
\hline \multirow[t]{5}{*}{$\mathrm{CBM76} 6_{\mathrm{RfGH} 44}$} & Xyloglucan & $1.1( \pm 0.0) \times 10^{6}$ \\
\hline & Glucomannan & $3.8( \pm 0.2) \times 10^{4}$ \\
\hline & $\beta$-Glucan & $1.2( \pm 0.1) \times 10^{4}$ \\
\hline & HEC & $2.6( \pm 0.1) \times 10^{4}$ \\
\hline & XXXG & $1.6( \pm 0.2) \times 10^{4}$ \\
\hline \multirow[t]{9}{*}{$\mathrm{CBM} 77_{\mathrm{RfPL} 1 / 9}$} & Lime Pectin DE $11 \%$ & $1.8( \pm 0.2) \times 10^{5}$ \\
\hline & GENU pectin $\mathrm{Cl}-114$ & $4.2( \pm 0.2) \times 10^{5}$ \\
\hline & PGA from orange & $1.2( \pm 0.02) \times 10$ \\
\hline & PGA from orange + EDTA & $1.1( \pm 0.0) \times 10^{4}$ \\
\hline & Pectin from citrus DE $30 \%$ & $1.1( \pm 0.0) \times 10^{4}$ \\
\hline & Pectin from citrus DE $60 \%$ & Binding weak* \\
\hline & Pectin from citrus $D E \geq 80 \%$ & No binding \\
\hline & GalA DP3/DP4 & Binding weak* \\
\hline & GalA DP7/DP8 & $1.2( \pm 0.2) \times 10^{5}$ \\
\hline \multirow[t]{8}{*}{ CBM78 ${ }_{\text {RfGH5 }}$} & Xyloglucan & $1.4( \pm 0.1) \times 10^{5}$ \\
\hline & $\beta$-Glucan & $2.4( \pm 0.4) \times 10^{3}$ \\
\hline & $\mathrm{HEC}$ & $2.1( \pm 0.2) \times 10^{4}$ \\
\hline & $X X X G$ & $3.0( \pm 0.7) \times 10^{3}$ \\
\hline & Cellohexaose & $1.7( \pm 0.1) \times 10^{4}$ \\
\hline & Cellopentaose & $8.6( \pm 0.2) \times 10^{3}$ \\
\hline & Cellotetraose & Binding weak* \\
\hline & $\mathrm{RC}$ & No binding \\
\hline \multirow{8}{*}{ CBM79-1 $1_{\text {RfGH }}$} & Xyloglucan & $1.0( \pm 0.2) \times 10^{4}$ \\
\hline & $\beta$-Glucan & $4.0( \pm 0.2) \times 10^{4}$ \\
\hline & HEC & $7.8( \pm 0.5) \times 10^{4}$ \\
\hline & XXXG & No binding \\
\hline & Cellohexaose & $4.9( \pm 0.9) \times 10^{3}$ \\
\hline & Cellopentaose & $7.0( \pm 0.3) \times 10^{3}$ \\
\hline & Cellotetraose & $4.2( \pm 1.7) \times 10^{3}$ \\
\hline & $\mathrm{RC}$ & $4.8( \pm 0.2) \times 10^{4}$ \\
\hline \multirow[t]{12}{*}{ CBM80 ${ }_{\text {RfGH5-1/2 }}$} & Xyloglucan & $1.8( \pm 0.1) \times 10^{5}$ \\
\hline & Glucomannan & $5.8( \pm 0.5) \times 10^{4}$ \\
\hline & Galactomannan & $4.5( \pm 0.2) \times 10^{4}$ \\
\hline & $\beta$-Glucan & $2.2( \pm 0.2) \times 10^{4}$ \\
\hline & HEC & $3.6( \pm 0.1) \times 10^{3}$ \\
\hline & $X X X G$ & No binding \\
\hline & Mannohexaose & $4.1( \pm 0.9) \times 10^{4}$ \\
\hline & Mannopentaose & $2.9( \pm 0.4) \times 10^{4}$ \\
\hline & Mannotetraose & $1.8( \pm 0.2) \times 10^{3}$ \\
\hline & Cellohexaose & $1.7( \pm 0.2) \times 10^{4}$ \\
\hline & Cellopentaose & $8.5( \pm 0.7) \times 10^{3}$ \\
\hline & Cellotetraose & No binding \\
\hline
\end{tabular}

Affinities were determined by isothermal titration calorimetry. The thermodynamics of ligand binding are reported in Tables S2-S7. HEC, hydroxyethyl cellulose; RC, regenerated cellulose; XXXG, xyloglucan heptasaccharide.

*Binding too weak to quantify by ITC.

core pectin binding residues in CBM77 $7_{\text {RfPL1/9 }}\left(\right.$ Lys $^{1092}$, Lys $^{1107}$, or Lys ${ }^{1162}$ ) were invariant (Fig. S2). Thus, pectin recognition seems to be a conserved feature of CBM77.

CBM78 ${ }_{\text {RFG }}$ and CBM79-1 $1_{\text {RfGH }}$ are representatives of CBMs that bind $\beta$-glucans. CBM78 $8_{\mathrm{RfGH} 5}$ and $\mathrm{CBM} 79-1_{\mathrm{RfGH} 9}$ contain two $\beta$-sheets defined as 1 and 2, respectively, (Fig. S4). In both CBMs, $\beta$-sheet 2 forms a cleft in which aromatic residues are a dominant feature (Fig. 3). In CBM78 $8_{\text {RfGH5}}, \operatorname{Trp}^{496}, \operatorname{Trp}^{554}, \mathrm{Tyr}^{555}$, and Phe ${ }^{479}$ are aligned along the cleft whereas, in CBM79-1 $1_{\mathrm{RfGH} 9}, \operatorname{Tyr}^{563}, \operatorname{Trp}^{564}$, $\operatorname{Tyr}^{597}$, Trp ${ }^{666}$, and $\operatorname{Trp}^{607}$ form a twisted hydrophobic platform. These hydrophobic regions are predicted to comprise the glucan binding sites in the two CBMs (Fig. $3 A$ and $B$ ).
Mutagenesis confirmed the importance of the aromatic residues in ligand recognition in $\beta$-sheet 2 of the two proteins. Alanine substitution of $\operatorname{Trp}^{496}$ or $\operatorname{Trp}^{554}$ in CBM78 ${ }_{\text {RfGH5}}$, and $\operatorname{Trp}^{606}$ in CBM79-1 ${ }_{\text {RfGH9, }}$ which are conserved in the two CBM families (Fig. S2), resulted in complete loss of binding to all ligands (Tables S2 and S3). A significant feature of these mutagenesis experiments was that alanine substitutions of several residues modulated ligand specificity. With respect to $\mathrm{CBM} 78_{\mathrm{RfGH} 5}$, the mutants $\mathrm{F} 479 \mathrm{~A}$ and Y555A bound to xyloglucan, but not to barley $\beta$-glucan or HEC. The variant $\mathrm{Q} 552 \mathrm{~A}$ recognized xyloglucan and barley $\beta$-glucan, but not HEC (Table S2). The equivalent residue to $\mathrm{CBM} 78_{\mathrm{RfGH}} 5$ $\mathrm{Phe}^{479}$ is not aromatic in four members of family CBM78 (Fig. S2), suggesting that these proteins may bind to xyloglucan but not to other $\beta$-glucans. In CBM79- $1_{\mathrm{RfGH} 9}$, the mutants W564A and W607A retained affinity for barley $\beta$-glucan but did not bind xyloglucan (Table S3). Trp ${ }^{607}$ in CBM79-1 ${ }_{\mathrm{RfGH}}$ is replaced by glycine in two members of CBM79 (Fig. S2), suggesting that there are also differences in specificity within this family. To summarize, the mutagenesis data show that whereas core residues play a generic role in binding $\beta$-glucans, other amino acids in the two CBMs play distinct roles in ligand recognition, explaining why these proteins can bind to a range of $\beta$-glucans.

The topology of the ligand binding site of $\mathrm{CBM} 78_{\mathrm{RfGH}}$ and CBM79-1 $1_{\text {RfGH9 }}$ is very different even though the positions of the key glucan binding aromatic residues are conserved. In CBM78 ${ }_{\mathrm{RfGH} 5}$, the cleft is a narrow canyon-like structure. In CBM79-1 RfGH9 , however, the concave surface forms an unusually solvent exposed cleft or planar surface, with loops connecting $\beta$-strands 1 and 2 and $\beta$-strands 4 and 5 strongly contributing to the curved topology of $\beta$-sheet 2 (Fig. $3 A$ and $B$ ). These contrasting topologies reflect the orientation of two tryptophan residues that play a key role in ligand recognition. With respect to $\beta$-sheet 2 , these residues adopt a planar $\left(\operatorname{Trp}^{564}\right.$ and $\left.\operatorname{Trp}^{606}\right)$ or perpendicular $\left(\operatorname{Trp}^{496}\right.$ and $\operatorname{Trp}^{554}$ ) orientation in CBM79-1 $1_{\mathrm{RfGH}}$ and $\mathrm{CBM} 78_{\mathrm{RfGH} 5}$, respectively (Fig. $3 E$ ). In CBM7 $8_{\mathrm{RfGH} 5}, \beta$-sheet 2 contains two additional $\beta$-strands $(7,8)$, and $\mathrm{Il}^{522}$ from strand 7 stacks against the indole ring of $\operatorname{Trp}^{496}$, enabling the aromatic residue to adopt a perpendicular orientation. The $\mathrm{N} \varepsilon$ of $\operatorname{Trp}^{554}$ makes a polar contact with $\mathrm{O} \varepsilon 1$ of $\mathrm{Gln}^{552}$, which orientates the indole ring into its perpendicular conformation. In contrast, there are no steric constraints preventing $\operatorname{Trp}^{564}$ and $\operatorname{Trp}^{606}$ in CBM79-1 $1_{\mathrm{RfGH}}$ from making apolar planar interactions with the peptide chain of $\beta$-strands $4 / 7$ and $4 / 5$, respectively.

The planar topology of the binding cleft of CBM79- $1_{\text {RfGH }}$ indicates that this protein may interact with components of insoluble cellulose. The narrow binding cleft of CBM78 ${ }_{\text {RfGH5 }}$ points to a specificity for discrete cellulose chains, which occur rarely in insoluble cellulose. ITC and pull down assays showed that $\mathrm{CBM} 79-1_{\mathrm{RfGH} 9}$, but not $\mathrm{CBM} 78_{\mathrm{RfGH}_{5}}$, bound to regenerated (noncrystalline) insoluble cellulose (RC) (Fig. S1 $C$ and $D$ ). The inability of W564A and W606A to bind RC shows that recognition of the polysaccharide is in solvent exposed cleft (Fig. $\mathrm{S} 1 D$ ). The impact of the conformation adopted by conserved aromatic residues on CBM specificity is also apparent in family 2 CBMs that bind to cellulose or xylan (13). The differences in the specificity of $\beta$-glucan binding CBMs reported here provide a biological rationale for the evolution of a large number of CBMs that target these glycans. Within the context of a complex cellulosome structure, the myriad of $\beta$-glucan binding CBMs may act in synergy to bind different substructures of cellulose, obviating the need for a classical type A module that binds crystalline cellulose. CBM80 ${ }_{\text {RfGH5-1/2. }}$. The apo structure of CBM80 ${ }_{\mathrm{RfGH} 5-1 / 2}$ and in complex with mannohexaose and cellohexaose was solved to a resolution of $1.0 \AA, 1.4 \AA$, and $1.5 \AA$, respectively. The $\beta$-sheet 2 of CBM80 ${ }_{\text {RfGH5-1/2 }}$ presents a planar hydrophobic surface, through the approximately parallel orientation of $\operatorname{Trp}^{453}$ and $\operatorname{Trp}_{490}^{489}$. Distal to this planar surface is a third aromatic residue, Trp ${ }^{490}$, which is in a perpendicular orientation to the two other solvent-exposed tryptophan residues.

The mannohexaose-CBM80 ${ }_{\text {RfGH5-1/2 }}$ complex revealed electron density for mannohexaose (Fig. S5 $A$ ) along the hydrophobic 

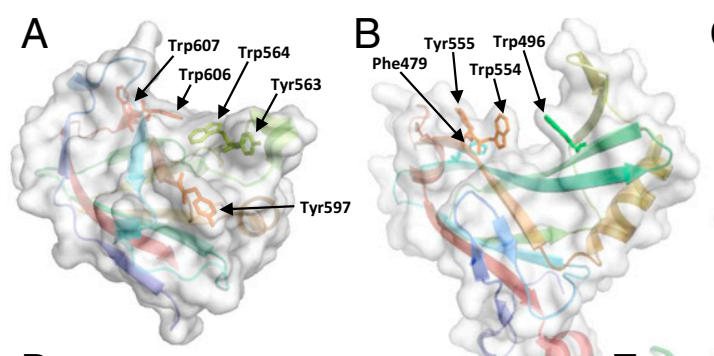

C

D

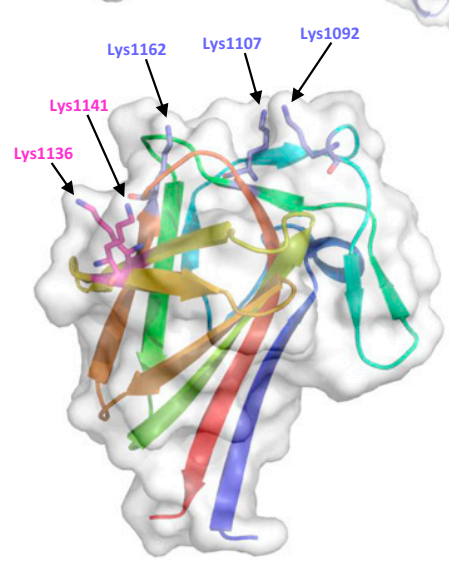

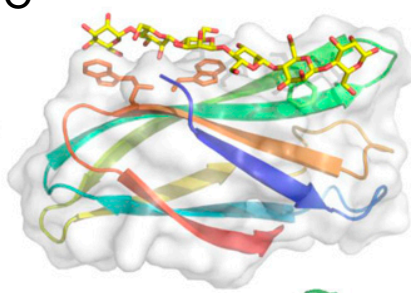

E

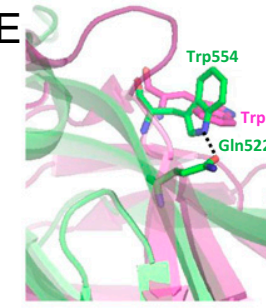

$\mathrm{F}$

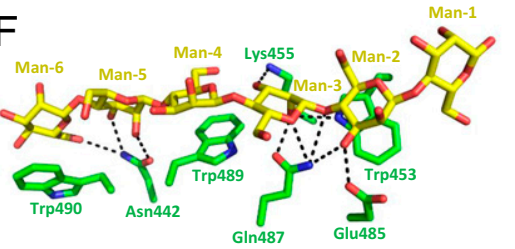

Fig. 3. Crystal structures of CBM79-1 $1_{\mathrm{RfGH}}, \mathrm{CBM78} 8_{\mathrm{RfGH} 5}$ $\mathrm{CBM}_{\mathrm{RfGH}-1 / 2}$, and $\mathrm{CBM} 77_{\mathrm{RfPL} 1 / 9}$. $(A-D)$ Schematics of $\mathrm{CBM} 78_{\mathrm{RfGH} 5}, \mathrm{CBM} 79-1_{\mathrm{RfGH} 9}, \mathrm{CBM} 80_{\mathrm{RfGH} 5-1 / 2}$, and $\mathrm{CBM77} 7_{\mathrm{RfPL} 1 / 9}$, respectively, color ramped from $\mathrm{N}$ terminus (blue) to $C$ terminus (red), embedded in the surface representation of the proteins. The aromatic (CBM78 RfGH5$_{1}$ CBM79-1 RfGH9, CBM80 $_{\text {RfGH5-1/2 }}$ ) or basic $\left(\mathrm{CBM} 77_{\mathrm{RfPL} 1 / 9}\right)$ residues that contribute to ligand recognition are shown in stick format and identified in $A, B$, and $D$, while mannose carbons are colored yellow in $C$. (E) An overlay of $C B M 78_{\mathrm{RfGH}_{5}}$ (green) and

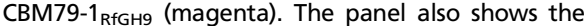
residues that interact with the aromatic amino acids in $\mathrm{CBM}_{78_{\mathrm{RfGH}} \text {. }}(F)$ The amino acids (carbons colored green) in the structure of $\mathrm{CBM} 80_{\mathrm{RfGH}-1 / 2}$ that make polar (indicated by black dashed lines) or apolar interactions with mannohexaose (carbons colored yellow). The ligands are labeled from the reducing end (i.e., Man-1). This figure and Fig. S5 were prepared using PyMol. surface of $\beta$-sheet 2 . All of the pyranoside sugars were in the undistorted ${ }^{4} \mathrm{C}_{1}$ chair conformation. The three solvent-exposed aromatic residues $\left(\operatorname{Trp}^{453}, \operatorname{Trp}^{489}\right.$, and $\operatorname{Trp}^{490}$ ) interact with the $\alpha$-face of the pyranose rings of the mannoside residues 2,4 , and 6 , respectively. The minimum ligand required to harness the binding energy from all three aromatics is a pentasaccharide, consistent with the ITC binding data (Table 1). There are few direct hydrogen bonds between $\mathrm{CBM} 80_{\mathrm{RfGH} 5-1 / 2}$ and mannohexaose (Fig. 3). Mannoside-1 (reducing end sugar) and mannoside-4 make no direct hydrogen bonds to the CBM. The $\mathrm{O} 3$ of mannoside-2 forms hydrogen bonds with $\mathrm{O} \varepsilon 1$ of $\mathrm{Glu}^{485}$, and $\mathrm{N} \varepsilon 2$ of $\mathrm{Gln}^{487}$ makes a polar contact with $\mathrm{O} 3$ and $\mathrm{O} 4$. Mannoside-3 interacts with the protein through polar contacts between $\mathrm{O} 2$ and both $\mathrm{O} \varepsilon 1$ of $\mathrm{Gln}{ }^{487}$ and N\&1 of $\operatorname{Trp}^{489}$, and between O3 and N 11 of Lys ${ }^{455}$, whereas the endocyclic oxygen accepts a hydrogen from N 22 of $\mathrm{Gln}^{487} . \mathrm{O} 2$ of mannoside- 5 makes a hydrogen bond with O 11 of $\mathrm{Asn}^{442}$ whereas N82 of the asparagine makes polar contacts with $\mathrm{O} 3$ of mannosides 5 and 6 , respectively. The $\mathrm{O} 6$ is solvent-exposed at mannosides- 1 to -5 , and thus CBM80 ${ }_{\mathrm{RfGH}-1 / 2}$ is able to recognize the backbone of galactomannans in which the mannan backbone is decorated with $\alpha-1,6-\mathrm{D}-\mathrm{galactose}$ side chains. The structure of CBM80 ${ }_{\text {RfGH5-1/2 }}$ in complex with cellohexaose revealed electron density for only three glucose units, which were also modeled in their relaxed ${ }^{4} \mathrm{C}_{1}$ conformation (Fig. S5B). The cellulooligosaccharide and the corresponding region of mannohexaose made similar interactions with the CBM (Fig. S5C). The only differences were that $\mathrm{O} 2$ of glucoside-2 and glucoside- 3 interacted with $\mathrm{O} \varepsilon 1$ of $\mathrm{Glu}^{485}$ and N 1 of Lys ${ }^{455}$, respectively, whereas Gln ${ }^{487}$ made three fewer polar contacts with the gluco-configured ligand, compared with the mannooligosaccharide.

AGE showed that the mutants K455A, E485A, and Q487A of CBM80 ${ }_{\text {RfGH5}}$ retained WT affinity for all of the ligands tested, which suggests that the predicted polar interactions between the protein and $\beta$-glycans have very little influence on affinity. These data are unusual among type B CBMs where direct polar interactions generally make an important contribution to ligand recognition $(14,15)$. The data, however, are consistent with CfCBM2b-1, in which affinity is dominated by two tryptophans (16). Although removal of polar interactions greatly reduced the enthalpy of binding, because of enthalpy-entropy compensation, the mutations did not influence $K_{\mathrm{A}}$ values, which likely reflect the exposed binding site presented by this protein. $\mathrm{CBM} 80_{\mathrm{RfGH} 5-1 / 2}$ also contains a highly exposed ligand binding site, and thus the retention of WT affinity in the polar mutants may also result from enthalpy-entropy compensation. The distinguishing feature of glucose and mannose is the stereochemistry at $\mathrm{O} 2$, which adopts an equatorial or axial conformation, respectively. The observation that Q487A, K455A, and E457A substitutions did not influence affinity for $\beta$-glucans or $\beta$-mannans indicates that $\mathrm{O} 2$ is not a significant specificity determinant for these ligands, which explains why CBM80 RfGH5 is able to bind to both cellulose and $\beta$-mannan. Examples of CBMs that recognize both $\beta-1,4$-glucans and $\beta-1,4-$ mannans are found in families CBM16 (17) and CBM29 (CBM29-2) (18). In both proteins, residues that interact with $\mathrm{O} 2$ can make hydrogen bonds with the hydroxyl in either its axial or equatorial conformation. As discussed above, this finding is in contrast to $\mathrm{CBM} 80_{\mathrm{RfGH} 5-1 / 2}$, where polar interactions with its ligands do not seem to contribute to affinity or specificity.

A key discriminator of ligand specificity is likely to be the location of the three aromatic residues in the binding cleft that exhibit specificity for polysaccharide chains displaying a "twisted" conformation. This conformation has been shown for cellohexaose in solution (19) and when bound to other CBMs (20). Given that CBM80 ${ }_{\text {RfGH5-1/2 }}$ does not target the O6 groups in hexasaccharides, it is perhaps surprising that the CBM does not bind xylan. This finding likely reflects a binding cleft that is optimized to bind a twisted $\beta-1,4$-glycan chain and that is unable to accommodate the conformation adopted by xylan, a helical glycan with a threefold screw axis.

The importance of the surface tryptophan residues in ligand recognition is revealed by the complete abrogation of binding to $\beta$-glucans and $\beta$-mannans when $\operatorname{Trp}^{453}$ and $\operatorname{Trp}^{489}$, which stack against mannoside and glucoside residues 2 and 4, were substituted with alanine (Table S4). The mutant W490A retained the capacity to bind $\beta-1,4$-mannans, albeit with a 10 -fold reduction in $K_{\mathrm{A}}$, but displayed no affinity for the $\beta$-glucans (Table S4). CBMs that bind to extensive $\beta-1,4$-glycans typically contain three aromatic residues that make apolar interactions with sugars $n, n+2$ and $n+4$ (18). Alanine substitution of any of these aromatic residues generally leads to a substantial reduction and often complete abrogation of binding (21). It is unique, therefore, to observe a differential effect on glycan recognition when one of these aromatic residues is substituted with alanine. It is not obvious why the W490A mutant introduced selectivity for 
manno-configured ligands. The crystal structure of the mutant revealed no significant conformational changes with an rmsd of $0.7 \AA$ compared with the WT CBM (Fig. S5D). A notable feature of $\operatorname{Trp}^{490}$ is that the side chain is orientated $\sim 90^{\circ}$ compared with the other two surface tryptophan residues. We speculate that $\operatorname{Trp}^{490}$ contributes less binding energy for mannan compared with glucan because the manno-configured ligand is required to adopt nonoptimal conformations to access the distal aromatic residue. Thus, substitution of $\operatorname{Trp}^{490}$ has less impact on mannan binding than $\beta$-1,4-glucan recognition, which would suggest that, although both mannooligosaccharides and cellulooligosaccharides display a twisted conformation when bound to a variety of CBMs, their minimum energy conformations are not identical.

\section{Conclusion}

In the last decade, the availability of genomic/metagenomic data has increased exponentially. It is apparent that HTPMs need to be developed to understand the biological and biotechnological significance of this explosion in sequence information. This study explored the use of microarray technology combined with HTP protein production to explore the function of the $177 R$. flavefaciens UNKs that constitute $50 \%$ of the subunits of the most complex cellulosome described to date. The data revealed six previously unidentified CBM families, of which five target $\beta$-glucans and/or $\beta$-mannan, and one recognizes homogalacturonan. Structural data, in addition to revealing the importance of basic residues in calcium-independent pectin recognition, showed how the conformation of conserved aromatic residues can have a profound influence on the topology of the substrate binding cleft and consequently influence specificity.

To conclude, the data reported here reveal an unprecedented expansion in glycan recognition by the cellulosomes of rumen bacteria specialized in PCW degradation. This finding indicates

1. Gilbert HJ (2010) The biochemistry and structural biology of plant cell wall deconstruction. Plant Physiol 153(2):444-455.

2. Demain AL, Newcomb M, Wu JH (2005) Cellulase, clostridia, and ethanol. Microbiol Mol Biol Rev 69(1):124-154.

3. Gilbert HJ, Knox JP, Boraston AB (2013) Advances in understanding the molecular basis of plant cell wall polysaccharide recognition by carbohydrate-binding modules. Curr Opin Struct Biol 23(5):669-677.

4. Lombard V, Golaconda Ramulu H, Drula E, Coutinho PM, Henrissat B (2014) The carbohydrate-active enzymes database (CAZy) in 2013. Nucleic Acids Res 42(Database issue):D490-D495

5. Bayer EA, Belaich JP, Shoham Y, Lamed R (2004) The cellulosomes: Multienzyme machines for degradation of plant cell wall polysaccharides. Annu Rev Microbiol 58:521-554.

6. Rincon MT, et al. (2010) Abundance and diversity of dockerin-containing proteins in the fiber-degrading rumen bacterium, Ruminococcus flavefaciens FD-1. PLoS One 5(8):e12476.

7. Pedersen $\mathrm{HL}$, et al. (2012) Versatile high resolution oligosaccharide microarrays for plant glycobiology and cell wall research. J Biol Chem 287(47):39429-39438.

8. Mewis K, Lenfant N, Lombard V, Henrissat B (2016) Dividing the large glycoside hy drolase family 43 into subfamilies: A motivation for detailed enzyme characterization. Appl Environ Microbiol 82(6):1686-1692.

9. Yoshida S, Hespen CW, Beverly RL, Mackie RI, Cann IK (2010) Domain analysis of a modular alpha-L-Arabinofuranosidase with a unique carbohydrate binding strategy from the fiber-degrading bacterium Fibrobacter succinogenes S85. J Bacterio/ 192(20): 5424-5436.

10. Jia Z, Qin Q, Darvill AG, York WS (2003) Structure of the xyloglucan produced by suspension-cultured tomato cells. Carbohydr Res 338(11):1197-1208.

11. Abbott DW, Hrynuik S, Boraston AB (2007) Identification and characterization of a novel periplasmic polygalacturonic acid binding protein from Yersinia enterolitica. J Mol Biol 367(4):1023-1033.

12. Hileman RE, Fromm JR, Weiler JM, Linhardt RJ (1998) Glycosaminoglycan-protein interactions: Definition of consensus sites in glycosaminoglycan binding proteins. BioEssays 20(2):156-167.

13. Simpson PJ, Xie H, Bolam DN, Gilbert HJ, Williamson MP (2000) The structural basis for the ligand specificity of family 2 carbohydrate-binding modules. J Biol Chem 275(52): 41137-41142.

14. Pell G, et al. (2003) Importance of hydrophobic and polar residues in ligand binding in the family 15 carbohydrate-binding module from Cellvibrio japonicus Xyn10C Biochemistry 42(31):9316-9323.

15. Su $X$, et al. (2010) Mutational insights into the roles of amino acid residues in ligand binding for two closely related family 16 carbohydrate binding modules. $J$ Biol Chem 285(45):34665-34676. that, in highly competitive ecological niches, where complex carbohydrates are used as a major carbon source, enzyme-substrate targeting through the function of CBMs plays a critical role in substrate acquisition and thus organism survival.

\section{Materials and Methods}

Cloning, Expression, Site-Directed Mutagenesis, and Purification of Cellulosomal UNKs. The genes encoding 177 cellulosomal UNKs from $R$. flavefaciens were cloned into Escherichia coli expression vectors. Details of the cloning strategies, site-directed mutagenesis, and the purification of the recombinant proteins are described in SI Materials and Methods. The sequences and molecular architecture of the proteins are provided in Fig. S6.

Glycan Binding Assays. The binding of CBMs to carbohydrate ligands (Fig. S7) was screened using carbohydrate microarrays printed on nitrocellulose and AGE. The binding of selected CBMs to their ligands was quantified by ITC and insoluble ligands by pull down experiments and are described in detail in SI Materials and Methods.

Crystallization, Data Collection, and Structure Solution. The structures of CBM78 ${ }_{\mathrm{RfGH}}, \mathrm{CBM79}-1_{\mathrm{RfGH}}$, and $\mathrm{CBM} 80_{\mathrm{RfGH} 5-1 / 2}$ were solved using singlewavelength anomalous diffraction (SAD) methods and selenomethionyl proteins, and CBM77 $7_{\text {RfPL1/9 }}$ was solved by sulfur SAD methods. Details of crystallization, data collection, and structure solution are given in SI Materials and Methods. Structure statistics are provided in Table S8.

ACKNOWLEDGMENTS. We thank Diamond Light Source (Harwell, UK) for access to beamlines 102, 104, and 104-1 (mx9948) that contributed to the results presented here, as well as the European Synchrotron Radiation Facility (Grenoble, France) and Soleil (Saint-Aubin, France) for data collection. This work was supported by the EU FP7 Programme under WallTraC Project Grant 263916, Biotechnology and Biological Sciences Research Council Project Grant BB/K020358/1, and BioStruct-X Grant 283570, by the Danish Strategic Research Council, and by the Danish Council for Independent Research, Technology and Production Sciences as part of GlycAct Project FI 10-093465 and Qren through Grant 30263.

16. Xie $\mathrm{H}$, et al. (2001) Role of hydrogen bonding in the interaction between a xylan binding module and xylan. Biochemistry 40(19):5700-5707.

17. Bae B, et al. (2008) Molecular basis for the selectivity and specificity of ligand recognition by the family 16 carbohydrate-binding modules from Thermoanaerobacterium polysaccharolyticum ManA. J Biol Chem 283(18):12415-12425.

18. Charnock SJ, et al. (2002) Promiscuity in ligand-binding: The three-dimensional structure of a Piromyces carbohydrate-binding module, CBM29-2, in complex with cello- and mannohexaose. Proc Natl Acad Sci USA 99(22):14077-14082.

19. Sugiyama H, Hisamichi K, Usui T, Sakai K, Ishiyama J (2000) A study of the conformation of beta-1,4-linked glucose oligomers, cellobiose to cellohexaose, in solution. $J$ Mol Struct 556(3):173-177.

20. Boraston $A B$, et al. (2002) Differential oligosaccharide recognition by evolutionarilyrelated beta-1,4 and beta-1,3 glucan-binding modules. J Mol Biol 319(5):1143-1156.

21. Nagy $T$, et al. (1998) All three surface tryptophans in Type lla cellulose binding domains play a pivotal role in binding both soluble and insoluble ligands. FEBS Lett 429(3): 312-316.

22. Boraston $A B$ (2005) The interaction of carbohydrate-binding modules with insoluble non-crystalline cellulose is enthalpically driven. Biochem $J$ 385(Pt 2):479-484.

23. Charnock SJ, et al. (2000) The X6 "thermostabilizing" domains of xylanases are carbohydrate-binding modules: Structure and biochemistry of the Clostridium thermocellum X6b domain. Biochemistry 39(17):5013-5021.

24. Kabsch W (2010) XDS. Acta Crystallogr D Biol Crystallogr 66(Pt 2):125-132.

25. Evans PR, Murshudov GN (2013) How good are my data and what is the resolution? Acta Crystallogr D Biol Crystallogr 69(Pt 7):1204-1214.

26. Terwilliger TC, et al. (2009) Decision-making in structure solution using Bayesian estimates of map quality: The PHENIX AutoSol wizard. Acta Crystallogr D Biol Crystallogr 65(Pt 6):582-601.

27. McCoy AJ, et al. (2007) Phaser crystallographic software. J App/ Cryst 40(Pt 4):658-674.

28. Sheldrick GM (2010) Experimental phasing with SHELXC/D/E: Combining chain tracing with density modification. Acta Crystallogr D Biol Crystallogr 66(Pt 4):479-485.

29. Emsley P, Lohkamp B, Scott WG, Cowtan K (2010) Features and development of Coot. Acta Crystallogr D Biol Crystallogr 66(Pt 4):486-501.

30. Murshudov GN, et al. (2011) REFMAC5 for the refinement of macromolecular crystal structures. Acta Crystallogr D Biol Crystallogr 67(Pt 4):355-367.

31. Winn MD, et al. (2011) Overview of the CCP4 suite and current developments. Acta Crystallogr D Bio/ Crystallogr 67(Pt 4):235-242.

32. Chen VB, et al. (2010) MolProbity: All-atom structure validation for macromolecular crystallography. Acta Crystallogr D Biol Crystallogr 66(Pt 1):12-21.

33. Correia MA, et al. (2011) Structure and function of an arabinoxylan-specific xylanase. J Biol Chem 286(25):22510-22520. 